\title{
Is bone loss after gastric bypass surgery associated with the extent of weight loss?
}

\author{
Sue A Shapses \\ Associate Professor and Director of the New Jersey Obesity Group, Department of Nutritional \\ Science, Rutgers University, New Brunswick, NJ, USA.
}

\section{Summary}

This Practice Point commentary discusses a prospective study by Fleisher et al. that showed bone loss in response to extreme weight loss in 23 patients following Roux-en-Y gastric bypass surgery. The decline in BMD at the hip, but not at the spine or distal radius, was found to be proportional to the extent of weight loss. Although dietary calcium and vitamin D intake markedly increased after surgery, serum 25 hydroxyvitamin $\mathrm{D}$, serum parathyroid hormone and urinary calcium levels remained at presurgery levels. Here, I highlight concerns about bone loss associated with severe weight loss, discuss mechanisms that might alter bone regulation during caloric restriction, and consider how diet might be used to prevent osteoporosis after weight-loss surgery.

\section{Keywords}

bone; gastric bypass surgery; parathyroid hormone; vitamin D; weight loss

\section{COMMENTARY}

Gastric bypass surgery as a treatment for obesity is increasing in prevalence, partly because of the development of safe surgical techniques associated with a reduced incidence of postoperative complications. The popularity of such procedures is also fuelled by the knowledge that substantial weight loss reduces comorbidities. However, severe obesity is associated with subtle abnormalities in bone-regulating hormones, including low serum levels of vitamin D and secondary hyperparathyroidism. Furthermore, patients may be at risk of low BMD after gastric bypass surgery because of reduced calcium absorption and inadequate intakes of calcium, vitamin D or other nutrients. Although gastric bypass surgery reduces comorbidities, the long-term consequences of extreme weight loss on bone are unclear.

Fleisher et $_{\text {al. }}{ }^{2}$ examined bone loss in 23 patients (mean BMI $47 \mathrm{~kg} / \mathrm{m}^{2}$ ) before and 1 year after Roux-en-Y gastric bypass. At 12 months, mean participants' weight loss was $45 \mathrm{~kg}$ and

(C) 2009 Macmillan Publishers Limited. All rights reserved

Correspondence Department of Nutritional Sciences Rutgers University 96 Lipman Drive New Brunswick NJ 08901-8525 USA shapses@aesop.rutgers.edu.

Competing interests The author declared an association with the following company: Bayer. See the article online for full details of the relationship. 
their levels of bone turnover markers had increased. BMD had decreased at the femoral neck (9.2\%) and total hip (8.0\%), but was unchanged at the lumbar spine and distal radius. Calcium-regulating hormones generally remained at baseline levels despite a marked increase in dietary calcium and vitamin D after surgery, although transient changes in these hormones occurred 3 months after surgery.

The findings of Fleisher et $a l .{ }^{2}$ support those of a prospective study by Coates $e t a l .{ }^{3}$ that showed a 7.8\% BMD loss at the total hip associated with a $37 \mathrm{~kg}$ weight reduction 9 months after gastric bypass surgery. Fleisher and colleagues' findings are also consistent with those of a study that examined patients 3 years after gastric bypass surgery and found that hip BMD, but not lumbar spine BMD, decreased to values below those of weight-matched controls. ${ }^{4}$ By contrast, Coates et al. ${ }^{3}$ found a small decline in lumbar spine BMD after surgery.

In any case, BMD measurements by dual-energy X-ray absorptiometry are problematic in severely obese individuals because of the excess adipose tissue that overlies the bone and an increased prevalence of osteoarthritis. The precision of measured BMD changes during weight loss can also be compromised by changes in body size. ${ }^{5}$ To address possible inaccuracies, Fleisher et al. also calculated the apparent BMD (a volu-metric estimation of bone density) at the femoral neck; that showed a smaller, but still significant, BMD loss. ${ }^{2}$ Bone loss could alternatively be determined at a peripheral site where one would expect a smaller change in excess weight, such as the distal radius; interestingly, neither Fleisher $e t$ $a l$. nor Coates et al. found bone loss at this site. However, the radius is often associated with high measurement variability. Bone loss at the hip, but not the spine, has also been observed with moderate weight loss. ${ }^{5}$ This pattern of loss might be related to the effects of elevated parathyroid hormone (PTH) levels, which cause more loss at cortical than trabecular bone sites $;{ }^{6}$ alternatively, weight-bearing sites, for example the hip, might be more vulnerable to the effects of weight reduction than other sites, such as the lumbar spine and radius.

Compared with participants in the study by Fleisher $e t a l, 2$ the 42 women in the study by Carrasco et al. ${ }^{7}$ lost less weight (39 kg) but experienced greater declines in BMD $(10.5 \%$ and $7.4 \%$ at the hip and spine, respectively). Many factors could explain these increased BMD losses, including the use of different instruments, the participants' lower initial body weight, or the use of an exclusively female population. Furthermore, participants in the study by Carrasco et al. had a lower daily dietary intake of vitamin D (400-800 IU) and calcium $(680-1,000 \mathrm{mg}) ;^{7}$ in the study by Fleisher et al., ${ }^{2}$ daily dietary intake of calcium was $1,300 \mathrm{mg}$ at baseline and 2,500 $\mathrm{mg}$ after surgery, whereas daily vitamin D intake was $700 \mathrm{IU}$ at baseline and 1,700 IU after surgery. Possibly, the high intake of these nutrients before and after surgery had a positive effect on PTH levels and bone. Fleisher et $_{\text {al. }}{ }^{2}$ participants had normal levels of serum PTH presurgery and postsurgery, whereas PTH has been shown to be high both presurgery and postsurgery when calcium and vitamin D intakes were lower at about $1 \mathrm{~g}$ and $440 \mathrm{IU}$, respectively. ${ }^{1}$ However, several studies ${ }^{1-3}$ showed that participants had serum 25 hydroxyvitamin D levels in the insufficient range (below 75 nmol/l) both before and after surgery, which would compromise calcium absorption and have important implications for other diseases. 
In the study by Fleisher et al., ${ }^{2}$ the high intakes of dietary calcium and vitamin D after gastric bypass surgery were unable to suppress the transient rise in serum PTH levels, raise levels of 25 hydroxyvitamin $\mathrm{D}_{2}$ or prevent bone loss. The findings were similar in studies in which participants' daily dietary intakes of these nutrients were comparatively low (935$1700 \mathrm{mg}$ calcium and 440-660 IU vitamin D). ${ }^{1,3,7}$ Severely obese patients typically have increased BMD and calcium absorption levels; consequently, clinicians should not hesitate to recommend weight loss in this population because of bone-health concerns. Conversely, preliminary evidence suggests that cortical BMD might be compromised in severely obese people; therefore, an improved understanding of the long-term consequences of weight loss on bone is crucial. A daily dietary intake of $1200-1500 \mathrm{mg}$ calcium and enough vitamin D to raise serum 25 hydroxyvitamin $\mathrm{D}$ levels to $\geq 75 / \mathrm{nmol} / \mathrm{L}$ should be recommended. At the present time, it is not clear whether very high intakes of vitamin $\mathrm{D}$ are associated with increased benefits on bone.

Dietary intake is markedly reduced in patients after surgery, so vitamin and mineral supplements must be recommended. Other modifiable risk factors, such as levels of activity, medications and other nutrients, might also have a role in bone health and should be considered in future studies that examine metabolic bone disease after gastric bypass surgery.

\section{References}

1. Riedt CS, et al. True fractional calcium absorption is decreased after Roux-en-Y gastric-bypass surgery. Obesity (Silver Spring). 2006; 14:1940-1948. [PubMed: 17135609]

2. Fleischer $\mathrm{J}$, et al. The decline in hip-bone density after gastric-bypass surgery is associated with extent of weight loss. J Clin Endocrinol Metab. 2008; 93:3735-3740. [PubMed: 18647809]

3. Coates PS, et al. Gastric-bypass surgery for morbid obesity leads to an increase in bone turnover and a decrease in bone mass. J Clin Endocrinol Metab. 2004; 89:1061-1065. [PubMed: 15001587]

4. Goode LR, et al. Bone and gastric-bypass surgery: effects of dietary calcium and vitamin D. Obes Res. 2004; 12:40-47. [PubMed: 14742841]

5. Shapses SA, Riedt C. Bone, body weight, and weight reduction: what are the concerns? J Nutr136. 2006:1453-1456.

6. Dempster DW, et al. On the mechanism of cancellous bone preservation in postmenopausal women with mild primary hyperparathyroidism. J Clin Endocrinol Metab. 1999; 84:1562-1566. [PubMed: 10323380]

7. Carrasco F, et al. Changes in bone mineral density, body composition and adiponectin levels in morbidly obese patients after bariatric surgery. Obes Surg. 2008 [doi:10.1007/s11695-008-9638-0]. 